\title{
La comunidad científica ante los usos de la ciencia: un análisis de la orientación de la actividad investigadora en el CSIC
}

\author{
Carmen Merchán-Hernández*; Oihana Valmaseda-Andia ** \\ * Universidad Pablo de Olavide, Facultad de Ciencias Sociales, Departamento de Sociología. Sevilla \\ Correo-e: cmerchan@upo.es | ORCID iD: https://orcid.org/0000-0002-9505-7573 \\ ** Universidad del País Vasco/Euskal Herriko Uniberstsitatea UPV/EHU. Facultad de Economía y Empresa. \\ Correo-e:oihana.valmaseda@ehu.eus | ORCID iD: https://orcid.org/0000-0001-9013-179X
}

Recibido: 06-11-2017; 2a versión: 13-02-2018; Aceptado: 26-02-2018.

Cómo citar este artículo/Citation: Merchán-Hernández, C.; Valmaseda-Andia, O. (2018). La comunidad científica ante los usos de la ciencia: un análisis de la orientación de la actividad investigadora en el CSIC. Revista Española de Documentación Científica, 41 (4): e215. https://doi.org/10.3989/redc.2018.4.1536

Resumen: Este trabajo analiza la orientación de la actividad investigadora de una comunidad científica y su relación con el entorno socioeconómico. Para ello toma como referencia teórica la distinción del "cuadrante de Pasteur" de Stokes que identifica cuatro tipos ideales de científicos en base a si orientan su actividad investigadora hacia la realización de contribuciones científicas para la compresión de los fenómenos y hechos o, por otro lado, al uso práctico y/o la aplicación del conocimiento fuera del ámbito científico. El análisis empírico se basa en una encuesta realizada a 1593 investigadores del Consejo Superior de Investigaciones Científicas (CSIC). Los resultados evidencian el predominio de un perfil de científicos que orientan su actividad fundamentalmente a la generación de conocimiento científico, sin implicarse tanto en su aplicación práctica. Pese a que la mayoría muestra una actitud favorable hacia la transferencia de conocimiento, en el desarrollo de su trabajo cotidiano predominan actividades orientadas a consolidarse en su carrera científica.

Palabras clave: comunidad científica; Organismo Público de Investigación; cuadrante de Pasteur; diagrama de Stokes; investigadores; actividad investigadora; transferencia de conocimiento.

\section{The scientific community and the uses of science: an analysis of the orientation of the research activity in the CSIC}

Abstract: This paper analyses the orientation of the research activities in a scientific community, and its relationships with its socio-economic environment. For this purpose, the Pasteur's quadrant of Stokes (1997) is used as theoretical background. This method identifies four ideal profiles of scientists based on whether they direct their research activity towards making scientific contributions aimed to the understanding of phenomena and facts or, on the other hand, to the practical use and/or application of knowledge outside the scientific sector. The empirical analysis is based on a survey of 1593 researchers from the Spanish National Research Council (CSIC). The results highlight the predominance of an scientists' profile that focus primarily their activity to the generation of scientific knowledge, without so much involvement in its practical application. Although most of them show a favorable attitude towards the knowledge transfer, in their daily work there is a predominance of activities which are aimed to the consolidation of their scientific career.

Keywords: scientific community; Public Research Organization; Pasteur's quadrant; Stokes diagram; researchers; research activity; knowledge transfer.

Copyright: (c) 2018 CSIC. Este es un artículo de acceso abierto distribuido bajo los términos de la licencia de uso y distribución Creative Commons Reconocimiento 4.0 Internacional (CC BY 4.0). 


\section{INTRODUCCIÓN}

Los organismos públicos de investigación (OPIs) juegan un papel importante en la competitividad empresarial y, en general, en las dinámicas socioeconómicas de los sistemas de I+D e innovación. Desde su surgimiento a finales del siglo XIX y comienzos del siglo $X X$, una de sus funciones tradicionales ha consistido en la prestación de servicios de conocimiento al ámbito socioeconómico. De hecho, en las últimas décadas se ha producido una notable evolución y diversificación de los organismos públicos de investigación en los países desarrollados con la misión expresa de mejorar la capacidad productiva de las empresas prestando asistencia técnica directa y realizando transferencia de tecnología de alto nivel (Leydesdorff y otros, 2002). Tanto es así que en algunos sistemas nacionales y regionales los OPIs se han convertido en uno de los principales productores de conocimiento científico. Para lograr este objetivo, acumulan una fuerza de trabajo especialmente bien formada, así como equipamientos e infraestructuras de alto nivel. En ocasiones, reúnen una parte importante de la capacidad de I+D en términos de investigadores, laboratorios o grandes infraestructuras científicas. Esto les ha llevado a convertirse en organizaciones estratégicas en las sociedades contemporáneas para una variada gama de funciones, tales como la formación de investigadores, la diseminación social de la ciencia, las relaciones internacionales o la orientación global de las políticas de I+D e innovación.

Por otra parte, los OPIs también han diversificado los vínculos que mantienen con las entidades privadas y públicas, así como los efectos que pueden tener en la competitividad empresarial (Valmaseda-Andia y otros, 2015). Más allá de la tradicional función de asistencia técnica, las posibilidades de interacción pueden ser muy variadas y trascienden la transferencia de conocimiento entendida de manera lineal (Perkmann y otros, 2013). En primer lugar, las relaciones suelen consistir cada vez más en la co-producción de conocimiento, a través de la participación conjunta en proyectos de investigación de envergadura que contribuyen a la conversión de los avances científicos en innovaciones radicales. En segundo lugar, existen flujos de conocimiento a través de la transferencia de personal en varias direcciones, así como colaboraciones informales y de difusión de conocimiento (MerchánHernández, 2012). En tercer lugar, los OPIs también destacan como agentes científicos clave en la comercialización de licencias de patentes a las empresas (Azagra-Caro, 2007). En ciertas ocasiones, asumen el liderazgo para comercializar los nuevos hallazgos promoviendo la creación de empresas. Finalmente, los tradicionales servicios técnicos también se han diversificado. Ahora es posible encontrar una alta gama de servicios intensivos en conocimiento que pueden contribuir a la difusión de procesos innovadores y al aumento de la capacidad de vigilancia tecnológica.

En este contexto, la revisión de la literatura empírica pone en evidencia la naturaleza multinivel de la interacción de la comunidad científica con el entorno socioeconómico, en el sentido de que este proceso viene determinado tanto por las características individuales de los investigadores, como por el contexto organizacional e institucional en el que trabajan ${ }^{1}$ (Fuentes y Dutrénit, 2012). Entre los determinantes referidos al contexto organizacional e institucional cabe destacar, fundamentalmente, los basados en la afiliación institucional de los investigadores (Perkmann y otros, 2013), la misión de la organización (Mowery y Sampat, 2005), la disciplina científica (Bekkers y BodasFreitas, 2008), el acceso a diferentes fuentes de financiación (Schartinger y otros, 2002), así como la orientación emprendedora de la universidad o la calidad de la investigación que realiza (Bellucci y Pennacchio, 2016).

Otros autores enfatizan la incidencia de los factores individuales, relacionados con las características de los investigadores. Por un lado, entre estos factores individuales cabe destacar la edad (Schartinger y otros, 2002), la experiencia previa en la interacción con el entorno socioeconómico (D'Este y Patel, 2007), el estatus académico y el género (Azagra-Caro, 2007). En suma, desde estos estudios empíricos se asume que los comportamientos más activos en cuanto a la interacción con el entorno socioeconómico se encuentran entre los investigadores de más avanzada edad, con mayor experiencia previa en transferencia de conocimiento y un elevado perfil de producción científica. Por otro lado, algunos estudios inciden en la importancia de los factores culturales (Etzkowitz, 1998; Lee, 1998 y 2000), especialmente relacionados con las actitudes, valores y percepción hacia la realización de actividades de transferencia de conocimiento al entorno socioeconómico (Bonaccorsi y Piccaluga, 1994; Merchán-Hernández y otros, 2015).

Es, precisamente, en el análisis actitudinal de la comunidad científica donde ponemos el foco del presente trabajo, introduciendo un matiz diferencial con respecto a las investigaciones existentes que consisten en abordar el objeto de estudio en un contexto particular: el análisis de la posición de los investigadores de un organismo público de investigación concreto, el Consejo Superior de Investigaciones Científicas (CSIC). El objetivo de este estudio es explorar la orientación de la actividad científica de los investigadores de este organismo 
público de investigación y su interacción con el entorno socioeconómico. Para facilitar la comprensión de este propósito, se toma como referencia teórica la distinción que realiza Donald E. Stokes (1997) sobre la actividad investigadora conocida como "el cuadrante de Pasteur". Así, Stokes (1997) plantea una nueva clasificación de las actividades de investigación e innovación, insertando las actividades de investigación entre dos coordenadas: la primera que dimensiona el avance del conocimiento y la segunda, su aplicación. Pese a que este diagrama de Stokes (1997) resulta conocido por la literatura especializada, existen limitados estudios que pongan en práctica esta propuesta para el análisis de determinadas organizaciones o programas gubernamentales dirigidos a la comunidad científica (Sousa y otros, 2009). Este estudio se realiza a través de una encuesta realizada a 1593 investigadores del Consejo Superior de Investigaciones Científicas. La fuente de datos empleada resulta novedosa y de un valor clave, tanto por su especificidad y tamaño, como por su carácter inédito. Los datos recogidos en esta encuesta permiten acceder y analizar por primera vez la actividad investigadora de los científicos del CSIC y su interacción con el entorno socioeconómico en el periodo 1999-2010.

El artículo se estructura como sigue. Primero, y tras este apartado introductorio, se establece el marco teórico a través de la revisión de la literatura que sintetiza las aportaciones más relevantes en torno al objeto de estudio. Segundo, se detalla la fuente de datos utilizada, se describe la muestra de estudio y se explica el método de análisis empleado. Tercero, se presentan los principales resultados alcanzados, que son contrastados con los obtenidos en otras investigaciones existentes. Cuarto, el trabajo finaliza con un apartado de conclusiones del estudio.

\section{MARCO TEÓRICO}

Recientemente, y por iniciativa en gran parte de los decisores públicos, muchas universidades y OPIs han incorporado en el quehacer de su actividad laboral el desarrollo de tareas de interacción con el entorno socioeconómico (Etzkowitz y Leydesdorff, 2000). Entre los principales motivos que conducen a las organizaciones científicas a colaborar con las empresas destacan la obtención del conocimiento necesario para testar la aplicación práctica de sus investigaciones y el acceso a tecnologías de producción (Perkmann y otros, 2013; Lee, 2000). La obtención de financiación adicional, tanto para el desarrollo de las investigaciones, como para la mejora del equipamiento científico, es otra de las razones que los investigadores sostienen como determinantes para la colaboración con el entorno empresarial (Geuna, 2001). Sin embargo, a pesar de las importantes ventajas que las organizaciones públicas de investigación obtienen de la interacción con empresas, las actitudes predominantes de los investigadores ejercen una influencia clave en esta interacción, dada la relativa libertad en el desarrollo de su actividad, y que históricamente los objetivos de la mayoría de las universidades y algunos OPIs no incluían la colaboración con el entorno socioeconómico como una de las misiones principales (Azagra Caro y Romero de Pablos, 2009). Por este motivo, en la práctica, viene resultando complicada la instauración de este tipo de interacción entre determinados perfiles de investigadores, especialmente cuando subyacen valores contrarios a estas actividades, bien por considerarse ilegítimas o porque se perciban como tareas que puedan restringir la libertad científica de los propios investigadores (D'Este y Perkmann, 2011). De igual forma, los problemas de apropiación del conocimiento generado entre los socios de la colaboración dificultan la compatibilidad de las motivaciones y objetivos de las empresas y las de los agentes científicos (Foray y Steinmueller, 2003).

La existencia de divergentes valores, normas y misiones, además de diferentes dinámicas de trabajo y entornos organizacionales en la comunidad científica frente al sector empresarial (Bonaccorsi y Piccaluga, 1994), explica, en parte, la actitud de los investigadores hacia la transferencia de conocimiento al entorno. En efecto, buena parte de los estudios empíricos que analizan, desde la perspectiva de la comunidad científica, la interacción con otros agentes del entorno, han tratado de dirimir si la actitud de los investigadores hacia el desarrollo de actividades de transferencia de conocimiento influye en el grado de implicación en las mismas por parte de la comunidad científica (Merchán-Hernández y otros, 2015).

En este sentido, un referente clave es el trabajo de Lee (1998) donde se examinan las actitudes de los académicos hacia la interacción con el entorno económico, con el fin de conocer los límites que ponen a la colaboración. El estudio está basado en una encuesta realizada a 986 profesores universitarios en 1994, representativa según disciplinas académicas y tipos de universidades. Los resultados evidencian que, en general, entre los profesores estadounidenses de la década de los noventa existía una actitud positiva hacia la participación de sus centros de investigación en el desarrollo económico local y regional, a través de la comercialización de la investigación académica y el asesoramiento a empresas privadas. No obstante, Lee (1998) advierte que estas actividades de comercialización no se han popularizado de forma 
general en la comunidad científica y, a menudo, se perciben como actividades que conllevan un riesgo considerable para el clásico ethos científico. Existen, por tanto, voces disidentes dentro de la comunidad científica que denuncian la posible privatización de la investigación y la consecuente corrosión de los valores universitarios, como la libertad para seleccionar la agenda de investigación, el libre flujo de la información y la persecución desinteresada del conocimiento.

Esta divergencia de actitudes se revela en distintos perfiles dentro de la comunidad científica (Lee, 1998). Los científicos de disciplinas aplicadas -como química, ingenierías, informática o ciencias de materiales- reflejan una actitud más favorable ante las diferentes alternativas de transferencia, frente a los científicos de disciplinas básicas o de ciencias sociales. En este sentido, está comúnmente aceptado en la literatura que la comunidad científica sigue principios inspirados en el tradicional ethos científico (Merton, 1973), tales como la libertad de publicar los resultados de la investigación, el prestigio profesional y la prevalencia de la investigación de calidad. Por el contrario, el entorno socioeconómico $y$, especialmente nos referimos al sector privado, está inspirado en otros tipos de normas, tales como la privacidad de conocimiento, la rentabilidad, la aplicación de la investigación y la mejora de la posición competitiva de las empresas (Etzkowitz, 1998). Además, también difieren los principios organizacionales de ambos tipos de ambientes: las organizaciones científicas mantienen una estructura rígida y burocrática, mientras que las empresas tienden a ser más flexibles a fin de fomentar la competencia y adaptarse a los cambios en el mercado. En consecuencia, los plazos de la investigación por parte de la comunidad científica tienden a ser más dilatados en el tiempo, frente a las empresas que tienen como objetivo resolver problemas en el corto plazo (Bonaccorsi y Piccaluga, 1994).

Sin embargo, esta tradicional atribución de un conjunto de valores específicos a la institución de la ciencia ha sido cuestionada por recientes estudios empíricos que evidencian cómo los investigadores se adaptan a las expectativas de su entorno organizacional y ajustan sus valores a estas nuevas exigencias si existen incentivos para ello. En esa línea, existe una extensa literatura referida al creciente auge de la orientación emprendedora en la comunidad científica (Tijssen, 2006). Algunos estudios señalan cómo la lógica institucional de los diferentes agentes ha venido convergiendo en lógicas complementarias, facilitando esto el desarrollo de actividades de interacción con el entorno socieconómico (Bjerregaard, 2010). Para el caso concreto del CSIC, el estudio de Azagra y Romero (2009) centrado en los determinantes institucionales de las patentes del CSIC concluye que el marco institucional de este organismo de investigación resulta eficaz para crear una cultura favorable a la patente. En este sentido, Etzkowitz (1998) sugirió la aparición, en algunos contextos, de una ética comercial entre los investigadores que se contrapone al tradicional ethos científico (Merton, 1973), y que difumina las diferencias entre la lógica académica y comercial (Owen-Smith, 2003). Consecuentemente, emerge la figura del científico emprendedor que compagina el hábito tradicional, de contribuir al conocimiento científico y publicar, con el hábito emprendedor basado en la búsqueda de resultados potencialmente comerciales.

Sin embargo, otros estudios reclaman que la emergencia del rol de científico emprendedor no es tan extendida como sugiere Etzkowitz (1998). Normalmente, los científicos orientan sus actividades atendiendo al sistema de recompensas de la ciencia, basado en la excelencia científica, mientras que el sector productivo se guía por el imperativo de producir resultados comercializables (Dasgupta y David, 1994). Por lo tanto, intervienen a la vez dos lógicas divergentes en la actitud de la comunidad científica. Los principales límites a esa interacción con el entorno socioeconómico, para los investigadores, radican en un complicado equilibrio entre sus preocupaciones por el mantenimiento de la viabilidad económica de las organizaciones en las que desarrollan su actividad y el impacto que la colaboración con las empresas puede causar sobre su libertad investigadora (Lee, 1998). Así, Lee (1998) insiste en la existencia de una frontera entre la comunidad científica y la industria, cuestión sobre la que resulta necesario centrar el debate para la búsqueda de un equilibrio, tanto desde el mundo académico, como el empresarial.

Este tipo de dilema puede ser más acuciante, y de plena actualidad en la agenda política, en entornos periféricos, caracterizados por un tejido empresarial que realiza un esfuerzo limitado en $I+D$ y con baja capacidad de absorción (Merchán-Hernández y otros, 2015). Este tipo de contextos pueden provocar que la comunidad científica distancie su actividad investigadora de las necesidades de las empresas, motivado fundamentalmente por el sistema de incentivos y evaluación de la producción científica basado en criterios de excelencia internacional, que suele estar lejos de la demanda empresarial local (Fernández-Esquinas y otros, 2006). Así, los científicos tratan de enfocar su actividad en el desarrollo de una investigación de excelencia internacional, que las empresas de este tipo de regiones no suelen tener capacidad de absorber. 
Con objeto de explorar la orientación de la actividad científica de los investigadores de un organismo público de investigación y su actitud hacia la interacción con el entorno socioeconómico, este trabajo toma como referencia el análisis de "cuadrantes de Pasteur" propuesto por Stokes (1997) en el que distingue dos dimensiones básicas: por un lado, la investigación orientada a la realización de contribuciones científicas para la compresión de los fenómenos y hechos y, por otro lado, la investigación orientada hacia el uso práctico y/o la aplicación del conocimiento fuera del ámbito científico o académico. Así, la primera dimensión se refiere al avance del conocimiento científico y la segunda, a su aplicación (Stokes, 1997). Tomando como referencia estos componentes de la actividad investigadora, Stokes construye una matriz en la que identifica tipos de organización de la ciencia dependiendo de que la investigación consista en la obtención de conocimiento fundamental de los fenómenos que se estudian, de que se concentre en la resolución de un problema práctico o de que genere una combinación de ambos resultados a la vez. Esta última la considera como situación ideal a la que tiende el paradigma de la ciencia contemporánea debido a la trascendencia de los descubrimientos. Los componentes de esta matriz consisten en cuadrantes de acuerdo con la presencia o ausencia de alguna de las condiciones anteriores. Stokes se refiere a estos cuadrantes con el nombre de un científico que puede considerarse como figura paradigmática de cada uno de los modelos típicos de ciencia.

El cuadrante 1 lo relaciona con el físico danés Niels Bohr, quien centró su investigación en el conocimiento puro sobre la estructura del átomo sin perseguir una implicación directa en la aplicación práctica, a pesar de la importancia que tuvo posteriormente. El cuadrante 2 lo asocia con el inventor americano Thomas Edison, quien se considera un paradigma de investigador que busca resolver problemas prácticos mediante el desarrollo de tecnologías innovadoras, que no expresó especial interés por el conocimiento fundamental y la realización de contribuciones a la ciencia en forma de publicaciones. En el cuadrante 3 sitúa como referente al químico francés Louis Pasteur debido a que reúne a la vez las características de ser una figura relevante de la ciencia de su tiempo, cuyos descubrimientos están guiados por la consideración de uso y son efectivos para resolver problemas prácticos. Por último, existe un cuadrante 4 al que Stokes considera "Is not empty" debido a que sus procedimientos no se ubican claramente en el ámbito de la investigación científico-tecnológica, o bien a que sus realizaciones no han sido fructíferas en alguno de dos los ámbitos. Este cuadrante engloba a aquellos investigadores que no obtienen ni co- nocimiento fundamental ni logran específicamente un uso concreto, incluyendo algunas experiencias en la historia contemporánea de la ciencia que se desarrollan para justificar creencias sin base científica, para construir carreras científicas basadas en contribuciones que no están demostradas o para justificar aplicaciones prácticas fallidas que no están sustentadas en evidencias científicas. Estos científicos, según Stokes, pueden ser importantes precursores de la actividad investigadora propia del cuadrante de Bohr, así como del cuadrante de Edison. Cita como ejemplo las investigaciones del ornitólogo estadounidense Roger Tory Peterson, si bien Stokes no le asignó nombre específico a este cuadrante (Stokes, 1997).

El modelo de Stokes tiene como referencia la política científica y tecnológica estadounidense, no obstante este marco de análisis sirve de inspiración para países que procuran trazar un camino sostenible en dirección hacia la innovación. El modelo de análisis que propone Stokes resulta intuitivo y se ha aplicado para el estudio de diferentes contextos: bien como guía para el desarrollo de la agenda de investigación (Simmons y otros, 2005; Price y Behrens, 2003); como modelo teórico para el análisis de la producción de conocimiento (Tsao y otros, 2008; Castro-Martínez y otros, 2016); para el análisis de la estrategia de financiación de cara a la formulación de políticas públicas de ciencia, tecnología e innovación (Brito Cruz, 2008); como modelo de discusión en el análisis de la producción de la investigación básica y aplicada (Balaram, 2008); o como modelo de análisis de los resultados de un programa de postgrado (Sousa y otros 2009).

Una explotación previa centrada en el CSIC y que toma como base el modelo de Stoke, es el trabajo de Castro-Martínez y otros (2016). En este trabajo los autores realizan un análisis general sobre la vinculación ciencia-sociedad y toman como base el modelo de análisis de Stoke para identificar los principales canales de transferencia de conocimiento de este organismo público de investigación. Con objeto de avanzar en esta línea, nuestro análisis aplica el modelo de análisis de Stoke para el estudio específico de los diferentes perfiles de investigadores dentro de una comunidad científica concreta en base a la orientación de su actividad investigadora y su actitud hacia la interacción con el entorno socioeconómico.

\section{METODOLOGÍA}

\subsection{Fuente de datos y muestra}

La fuente de datos empleada para definir la población objeto de estudio se ha basado en la in- 
formación proporcionada por el Departamento de Recursos Humanos del CSIC, extraída del Registro Interno del Personal Funcionario y Laboral del CSIC. Dicha fuente de datos ofrece información del personal del CSIC en la fecha de Noviembre del 2010. La población operativa de este estudio está formada por todos los investigadores del CSIC que tienen título de doctor y poseen capacidad para firmar un contrato y/o convenio con otra entidad pública/privada. Teniendo en cuenta estos criterios para definir la población objeto de estudio, se ha obtenido un universo formado por 4.275 investigadores.

Para la realización de esta encuesta se ha seguido una estrategia de recogida de datos multimé- todos, que combina la realización del cuestionario online, con el seguimiento y refuerzo a través de llamadas telefónicas de encuestadores a los investigadores. El resultado de este procedimiento ha dado lugar a un total de 1593 entrevistas, lo que supone un error muestral de $\pm 1,9 \%$ para un nivel de confianza del 95\%, muy por encima de la tasa habitual en este tipo de encuestas. Asimismo, se ha realizado una post-estratificación según disciplinas científicas y categoría profesional. En las siguientes tablas se presenta la distribución de la población de investigadores del CSIC y la distribución de los investigadores encuestados según su categoría profesional y área científica (Tabla I y II), así como la representatividad de la muestra del estudio según estas dos variables (Tabla III).

Tabla I. Distribución de la población de investigadores del CSIC por categorías profesional y áreas científicas

\begin{tabular}{|c|c|c|c|c|c|}
\hline & $\begin{array}{c}\text { Post } \\
\text { Doctoral }\end{array}$ & $\begin{array}{c}\text { Científico } \\
\text { Titular }\end{array}$ & $\begin{array}{c}\text { Investigador } \\
\text { Científico }\end{array}$ & $\begin{array}{c}\text { Profesor } \\
\text { Investigador }\end{array}$ & Total \\
\hline Biología y biomedicina & $5,0 \%$ & $5,4 \%$ & $4,2 \%$ & $3,6 \%$ & $18,2 \%$ \\
\hline Ciencia y tecnología de alimentos & $0,9 \%$ & $2,7 \%$ & $1,8 \%$ & $1,3 \%$ & $6,7 \%$ \\
\hline Ciencia y tecnología de materiales & $3,3 \%$ & $4,4 \%$ & $2,8 \%$ & $2,7 \%$ & $13,3 \%$ \\
\hline Ciencia y tecnologías físicas & $3,5 \%$ & $4,2 \%$ & $3,3 \%$ & $2,5 \%$ & $13,4 \%$ \\
\hline Ciencia y tecnologías químicas & $2,1 \%$ & $4,0 \%$ & $3,0 \%$ & $2,2 \%$ & $11,3 \%$ \\
\hline Ciencias agrarias & $1,1 \%$ & $4,0 \%$ & $2,7 \%$ & $1,9 \%$ & $9,7 \%$ \\
\hline Humanidades y ciencias sociales & $2,0 \%$ & $3,4 \%$ & $2,3 \%$ & $1,9 \%$ & $9,5 \%$ \\
\hline Recursos naturales & $6,1 \%$ & $5,0 \%$ & $4,2 \%$ & $2,5 \%$ & $17,8 \%$ \\
\hline Total & $24,0 \%$ & $33,1 \%$ & $24,4 \%$ & $18,5 \%$ & $100,0 \%$ \\
\hline
\end{tabular}

Tabla II. Distribución de los investigadores encuestados por categorías profesional y áreas científicas

\begin{tabular}{|c|c|c|c|c|c|}
\hline & $\begin{array}{c}\text { Post } \\
\text { Doctoral }\end{array}$ & $\begin{array}{l}\text { Científico } \\
\text { Titular }\end{array}$ & $\begin{array}{l}\text { Investigador } \\
\text { Científico }\end{array}$ & $\begin{array}{c}\text { Profesor } \\
\text { Investigador }\end{array}$ & Total \\
\hline Biología y biomedicina & $2,9 \%$ & $5,3 \%$ & $3,6 \%$ & $3,6 \%$ & $15,3 \%$ \\
\hline Ciencia y tecnología de alimentos & $0,5 \%$ & $3,5 \%$ & $2,4 \%$ & $1,6 \%$ & $8,0 \%$ \\
\hline Ciencia y tecnología de materiales & $2,5 \%$ & $4,8 \%$ & $2,8 \%$ & $2,6 \%$ & $12,8 \%$ \\
\hline Ciencia y tecnologías físicas & $2,7 \%$ & $4,7 \%$ & $3,4 \%$ & $2,2 \%$ & $13,0 \%$ \\
\hline Ciencia y tecnologías químicas & $1,8 \%$ & $4,8 \%$ & $4,0 \%$ & $2,5 \%$ & $13,1 \%$ \\
\hline Ciencias agrarias & $0,7 \%$ & $5,4 \%$ & $4,2 \%$ & $2,4 \%$ & $12,7 \%$ \\
\hline Humanidades y ciencias sociales & $1,9 \%$ & $2,4 \%$ & $2,0 \%$ & $1,3 \%$ & $7,7 \%$ \\
\hline Recursos naturales & $5,1 \%$ & $5,4 \%$ & $4,6 \%$ & $2,3 \%$ & $17,4 \%$ \\
\hline Total & $18,0 \%$ & $36,3 \%$ & $27,1 \%$ & $18,6 \%$ & $100,0 \%$ \\
\hline
\end{tabular}


Tabla III. Variación porcentual de la muestra real respecto a la población por categorías profesional y áreas científicas

\begin{tabular}{|c|c|c|c|c|c|}
\hline \multicolumn{6}{|l|}{ Muestra-Población } \\
\hline & $\begin{array}{c}\text { Post } \\
\text { Doctoral }\end{array}$ & $\begin{array}{c}\text { Científico } \\
\text { Titular }\end{array}$ & $\begin{array}{c}\text { Investigador } \\
\text { Científico }\end{array}$ & $\begin{array}{c}\text { Profesor } \\
\text { Investigador }\end{array}$ & Total \\
\hline Biología y biomedicina & $-2,1 \%$ & $-0,1 \%$ & $-0,6 \%$ & $0,0 \%$ & $-2,9 \%$ \\
\hline Ciencia y tecnología de alimentos & $-0,4 \%$ & $0,8 \%$ & $0,5 \%$ & $0,3 \%$ & $1,3 \%$ \\
\hline Ciencia y tecnología de materiales & $-0,7 \%$ & $0,4 \%$ & $0,0 \%$ & $-0,1 \%$ & $-0,5 \%$ \\
\hline Ciencia y tecnologías físicas & $-0,8 \%$ & $0,6 \%$ & $0,1 \%$ & $-0,3 \%$ & $-0,4 \%$ \\
\hline Ciencia y tecnologías químicas & $-0,4 \%$ & $0,8 \%$ & $1,0 \%$ & $0,3 \%$ & $1,8 \%$ \\
\hline Ciencias agrarias & $-0,4 \%$ & $1,4 \%$ & $1,5 \%$ & $0,5 \%$ & $3,0 \%$ \\
\hline Humanidades y ciencias sociales & $-0,1 \%$ & $-0,9 \%$ & $-0,3 \%$ & $-0,5 \%$ & $-1,9 \%$ \\
\hline Recursos naturales & $-1,0 \%$ & $0,4 \%$ & $0,4 \%$ & $-0,2 \%$ & $-0,4 \%$ \\
\hline Total & $-6,0 \%$ & $3,3 \%$ & $2,7 \%$ & $0,1 \%$ & \\
\hline
\end{tabular}

A partir de estas tres tablas se advierte que, en lo que respecta a las diferentes categorías profesionales, la proporción de investigadores post doctorales encuestados ha sido menor respecto a la población total de post doctorales en el CSIC. En cambio, las categorías con más volumen de investigadores en el CSIC, especialmente las de científicos titulares, investigadores científicos y profesores de investigación, sí han tenido una representatividad mayor en la encuesta. Según las incidencias recogidas en el trabajo de campo, esta menor representación de post doctorales se ha debido a dificultades de contacto telefónico, por encontrarse muchos en el extranjero. En lo que respecta a la distribución por áreas científicas, en general la muestra es representativa de la población total de investigadores.

\subsection{Estrategia de análisis}

La estructura de la encuesta incluía un bloque de preguntas generales que permitían identificar los estilos de trabajo y las características de los equipos, así como un bloque específico centrado en recoger información sobre las actitudes y valores hacia el trabajo de investigación, con especial atención al papel otorgado a la interacción con el entorno socioeconómico. Dentro de este bloque se recabó información sobre las dimensiones propuestas por Stokes (1997) con el fin de conocer la orientación de la actividad realizada por los investigadores del CSIC y su actitud hacia la interacción con el entorno socioeconómico. Para ello se incluyeron dos preguntas de escala de actitud tipo Likert en las que se consultaba a los investigadores en qué medida su actividad investigadora está inspirada por: 1) Realizar contribuciones científicas a la comprensión de fenómenos y hechos; y 2) El uso práctico y/o la aplicación de los conocimientos fuera del ámbito científico o académico. Así, la primera pregunta se refiere a la dimensión avance del conocimiento científico y, la segunda, a su aplicación (Stokes, 1997). Estas dos escalas de actitud se han utilizado como base para realizar un ejercicio de clasificación de la orientación profesional de los investigadores del CSIC.

Para construir la tipología se han clasificado como orientaciones claras las respuestas que se sitúan en los puntos superiores de cada escala, frente a las que se sitúan en tres puntos más bajos, debido a que la distribución de las respuestas en los valores más bajos es muy reducida. Es necesario tener en cuenta que los comportamientos ante una escala de actitud siempre deben ser tomados con precaución debido a que las respuestas arrastran cierto grado de "deseabilidad" y no reflejan necesariamente las actividades que en realidad llevan a cabo las personas. No obstante, el ejercicio permite obtener un mapa de los valores que guían el trabajo de los investigadores y establecer cuáles son los grupos predominantes.

Tomando los resultados obtenidos en estas dos preguntas generales, la estrategia de análisis seguida en este trabajo se compone de dos fases. Primero, se identifican los cuatro tipos ideales o perfiles de científicos propuestos por Stokes (1997), dependiendo de si el investigador en su actividad científica está buscando el conocimiento fundamental o no (Pregunta 1) y si está implicado o no con los usos prácticos de los resultados de investigación (Pregunta 2). Segundo, se caracterizan los cuatro perfiles de científicos según diferentes variables relevantes, teniendo en cuenta las con- 
diciones que afectan al trabajo de investigación en dos de sus vertientes.

De un lado, es necesario estudiar la base de creencias y actitudes de los investigadores respecto a su trabajo. La orientación profesional es especialmente importante en un colectivo caracterizado por realizar tareas con un alto componente vocacional, que dispone de un alto grado de especialización y experiencia que es muy difícil de controlar por organizaciones o individuos ajenos al área de problemas de investigación específico en el que se trabaja. De otro lado, las actitudes y la orientación profesional se desarrollan y moldean en una estructura de oportunidades formada por los recursos materiales y económicos y las condiciones de trabajo que afectan al desarrollo profesional. Más concretamente, los mecanismos de evaluación y promoción y las condiciones administrativas para llevar a cabo la transferencia son aspectos que pueden influir en las relaciones que se tienen con el entorno.

Por todo ello, el estudio considera varios aspectos que tienen que ver con la base de valores y actitudes respecto al trabajo de investigación y con las condiciones concretas que afectan a las actividades de transferencia. En concreto las variables tomadas para dicho análisis han sido cuatro: las áreas científicas en las que están especializados los distintos perfiles de científicos, la forma en que desarrolla su actividad investigadora -en base a la composición de los equipos de trabajo y la organización de su actividad laboral-, su actitud hacia la transferencia e interacción con el entorno socioeconómico y, por último, las motivaciones de los investigadores para interactuar con el entorno. Esto permite obtener un análisis preciso de la actividad investigadora y, a la vez, contrastar las conclusiones sugeridas por la literatura en el caso de la comunidad científica del CSIC. El tipo de análisis de datos se ha basado en análisis de correspondencia que ha permitido identificar si existe diferencias significativas y asociación entre los cuatro perfiles de científicos con las variables independientes antes señaladas.

\section{RESULTADOS Y DISCUSIÓN}

A través de las dos dimensiones generales propuestas por Stokes (1997) sobre la orientación de la actividad investigadora, los resultados obtenidos indican que casi la totalidad de los encuestados (80\%) manifiestan que su actividad científica está inspirada por la comprensión de los fenómenos y hechos. Por otra parte, el $68 \%$ manifiesta que la consideración del uso/aplicación de los conocimientos también es un aspecto importante en su investigación. Tal como se observa en la Tabla IV, tomando los resultados de estas dos dimensiones, se puede situar a los investigadores del CSIC entre los cuatro tipos de científicos descritos por Stokes (1997). A partir de estos resultados, caracterizamos el perfil general de investigadores que componen cada uno de estos tipos de científicos en base a cuatro variables de análisis: el área o disciplina científica, la forma en la que desarrollan su actividad investigadora, la actitud que tienen hacia la transferencia de conocimientos científicos y, por último, las motivaciones que tienen los investigadores para establecer relaciones de colaboración con otras entidades públicas o privadas.

En primer lugar, los resultados obtenidos, en líneas generales, advierten que más de la mitad de los investigadores del CSIC (58\%) se sitúan en el cuadrante de Bohr ya que se caracterizan fundamentalmente por orientar su actividad investigadora hacia la comprensión de los fenómenos y hechos, sin implicarse en la aplicación práctica de sus resultados. Esta orientación de la actividad investigadora más dirigida hacia la investigación básica y menos hacia la aplicación del conocimiento es el perfil más extendido en el CSIC, el cual se extiende de manera transversal a todas las disciplinas científicas y categorías profesionales (Tabla V y VI). No obstante, las áreas de biología y biomedicina y de recursos naturales destacan por tener una mayor proporción de investigadores que orienta su actividad investigadora en mayor grado hacia la investigación básica. Asimismo, re-

Tabla IV. Orientación de la actividad investigadora según cuadrantes de Stokes

\begin{tabular}{|c|c|c|c|c|c|c|}
\hline & \multicolumn{5}{|c|}{ Consideración del uso/aplicación de los conocimientos } \\
\hline & & & Baja & & Alta & Total \\
\hline \multirow{6}{*}{$\begin{array}{c}\text { Comprensión } \\
\text { de los } \\
\text { fenómenos y } \\
\text { hecho }\end{array}$} & \multirow{2}{*}{ Alta } & \multirow{2}{*}{$B O H R$} & 926 & \multirow{2}{*}{ PASTEUR } & 353 & 1279 \\
\hline & & & $58 \%$ & & $22 \%$ & $80 \%$ \\
\hline & \multirow{2}{*}{ Baja } & \multirow{2}{*}{$\begin{array}{l}\text { IS NOT } \\
\text { EMPTY }\end{array}$} & 159 & \multirow{2}{*}{ EDISON } & 155 & 314 \\
\hline & & & $10 \%$ & & $10 \%$ & $20 \%$ \\
\hline & \multirow{2}{*}{ Total } & & 1085 & & 508 & 1593 \\
\hline & & & $68 \%$ & & $32 \%$ & $100 \%$ \\
\hline
\end{tabular}


Tabla V. Cuadrantes de Stokes según Áreas Científicas

\begin{tabular}{|c|c|c|c|c|c|c|c|c|c|}
\hline & $\begin{array}{c}\text { Biología y } \\
\text { biomedicina }\end{array}$ & $\begin{array}{c}\text { Ciencia y } \\
\text { tecnología } \\
\text { de } \\
\text { alimentos }\end{array}$ & $\begin{array}{c}\text { Ciencia y } \\
\text { tecnología } \\
\text { de } \\
\text { materiales }\end{array}$ & $\begin{array}{l}\text { Ciencia y } \\
\text { tecnologías } \\
\text { físicas }\end{array}$ & $\begin{array}{c}\text { Ciencia y } \\
\text { tecnologías } \\
\text { químicas }\end{array}$ & $\begin{array}{l}\text { Ciencias } \\
\text { agrarias }\end{array}$ & $\begin{array}{l}\text { Humanidades y } \\
\text { ciencias sociales }\end{array}$ & $\begin{array}{l}\text { Recursos } \\
\text { naturales }\end{array}$ & Total \\
\hline $\begin{array}{l}\text { Is not } \\
\text { empty }\end{array}$ & $5 \%$ & $20 \%$ & $11 \%$ & $9 \%$ & $15 \%$ & $9 \%$ & $11 \%$ & $6 \%$ & $10 \%$ \\
\hline Edison & $2 \%$ & $13 \%$ & $14 \%$ & $17 \%$ & $12 \%$ & $12 \%$ & $7 \%$ & $4 \%$ & $10 \%$ \\
\hline Bohr & $70 \%$ & $42 \%$ & $55 \%$ & $58 \%$ & $47 \%$ & $57 \%$ & $57 \%$ & $67 \%$ & $58 \%$ \\
\hline Pasteur & $24 \%$ & $24 \%$ & $21 \%$ & $15 \%$ & $26 \%$ & $22 \%$ & $25 \%$ & $23 \%$ & $22 \%$ \\
\hline Total & $\begin{array}{c}244 \\
100 \%\end{array}$ & $\begin{array}{c}127 \\
100 \%\end{array}$ & $\begin{array}{c}204 \\
100 \%\end{array}$ & $\begin{array}{c}207 \\
100 \%\end{array}$ & $\begin{array}{c}209 \\
100 \%\end{array}$ & $\begin{array}{c}203 \\
100 \%\end{array}$ & $\begin{array}{c}122 \\
100 \%\end{array}$ & $\begin{array}{c}277 \\
100 \%\end{array}$ & 1593 \\
\hline
\end{tabular}

Nota: Los resultados se basan en pruebas bilaterales con un nivel de significación 0.05 .

Tabla VI. Cuadrantes de Stokes según Categoría Profesional

\begin{tabular}{|c|c|c|c|c|c|}
\hline & Postdoctoral & $\begin{array}{l}\text { Científico } \\
\text { Titular }\end{array}$ & $\begin{array}{l}\text { Investigador } \\
\text { Científico }\end{array}$ & $\begin{array}{c}\text { Profesor } \\
\text { Investigador }\end{array}$ & Total \\
\hline Is not empty & $13 \%$ & $11 \%$ & $9 \%$ & $6 \%$ & $10 \%$ \\
\hline Edison & $7 \%$ & $11 \%$ & $10 \%$ & $9 \%$ & $10 \%$ \\
\hline Bohr & $56 \%$ & $56 \%$ & $63 \%$ & $58 \%$ & $58 \%$ \\
\hline Pasteur & $24 \%$ & $21 \%$ & $19 \%$ & $27 \%$ & $22 \%$ \\
\hline Total & $\begin{array}{c}287 \\
100 \%\end{array}$ & $\begin{array}{c}579 \\
100 \%\end{array}$ & $\begin{array}{c}431 \\
100 \%\end{array}$ & $\begin{array}{c}296 \\
100 \%\end{array}$ & 1593 \\
\hline
\end{tabular}

Nota: Los resultados se basan en pruebas bilaterales con un nivel de significación 0.05 .

sulta más extendido este tipo de orientación de la investigación entre los perfiles más consolidados en la carrera académica, especialmente entre los investigadores científicos.

El segundo grupo de científicos más importante en el CSIC, formado por el $22 \%$ de los investigadores, se sitúan en el cuadrante conocido como Pasteur al orientar su actividad investigadora hacia la compresión de los fenómenos con el fin de resolver problemas prácticos y la aplicación de dichos conocimientos científicos. Este perfil también se extiende casi en la misma proporción entre las distintas áreas científicas, si bien cabe destacar una mayor incidencia entre los investigadores de ciencias y tecnologías químicas y de humanidades y ciencias sociales. Atendiendo a la categoría profesional de los investigadores, se observa que este modelo de investigación es más representativo en los dos extremos de la carrera académica, especialmente entre los profesores de investigación.

Por otra parte, sólo el $10 \%$ de los investigadores se caracterizan por orientar su actividad principalmente hacia la resolución de problemas prácticos (Cuadrante de Edison). En este cuadrante se sitúan especialmente investigadores de ciencias y tecnologías físicas y de materiales, así como científicos titulares, aunque las diferencias entre las categorías superiores es muy próxima. Por último, otro $10 \%$ de los investigadores se sitúan en el denominado cuadrante "Is not empty". En este cuadrante cabe destacar a los investigadores de ciencias y tecnologías de los alimentos y de químicas, así como, ya en menor medida, los investigadores de ciencias materiales y de humanidades y ciencias sociales. En lo referido a las categorías más destacadas en este cuadrante, cabe resaltar un mayor porcentaje de investigadores postdoctorales. No obstante, es conveniente resaltar que esta cantidad puede reflejar el comportamiento ante la escala y el procedimiento de consignación de las respuestas, y no necesariamente el que no otorguen importancia a los dos dimensiones citadas en el trabajo concreto que realizan. El ejercicio sirve, por tanto, para clasificar tendencias en las actitudes y no para observar comportamientos efectivos, aunque en los siguientes resultados se comprueba que esta clasificación suele ser consistente con las opiniones hacia la transferencia de conocimiento, con las actividades que se realizan con entidades del entorno socioeconómico y con aspectos fundamentales de la organización del trabajo de los investigadores.

En segundo lugar, se analiza la forma en que desarrollan su trabajo de investigación estos cien- 
tíficos a través del estudio de la composición de los equipos de investigación y el grado de importancia concedido a distintos aspectos del trabajo como investigador. De este modo, los resultados revelan que la gran mayoría de investigadores trabajan habitualmente dentro de un grupo de investigación estable (Figura 1). Tomando los distintos perfiles de científicos también se advierten diferencias significativas en las formas en que desarrollan su trabajo.

Pese a que la mayoría de los investigadores de los cuatro cuadrantes desarrollan su actividad dentro de un grupo de investigación estable, los investigadores situados en el cuadrante "Is not empty" destacan por este tipo de forma de trabajo. Resulta razonado que los investigadores que orientan su actividad hacia el desarrollo de investigaciones con el fin de publicar para el avance de sus carreras académicas, busquen para ello respaldo en un grupo de investigación consolidado, que les permita beneficiarse de su estabilidad y de su posible volumen de datos para orientar su actividad, fundamentalmente, hacia las publicaciones. Por otra parte, resulta significativo el porcentaje de investigadores que trabajan de forma más versátil entre los investigadores que orientan su actividad, principalmente, hacia la resolución de problemas prácticos (Cuadrante de Edison y de Pasteur), desarrollando su actividad en un equipo de varias personas de acuerdo con las necesidades del trabajo; mientras que entre los investigadores más orientados a la compresión de los fenómenos (Cuadrante de Bohr) es representativo el porcentaje de aquellos que desarrollan su actividad de manera individual, con colaboraciones puntuales.

Otro rasgo clave en la caracterización de la actividad investigadora es identificar el grado de importancia que conceden los científicos a las distintas tareas que engloba su trabajo. En este sentido, como se muestra en la Figura 2, los investigadores reconocen como los aspectos más importantes de su quehacer científico prácticas o valores que se encuentran más alineadas con los principios de la "ciencia académica tradicional" y que, por lo tanto, pueden generar tensiones con el proceso de colaboración con el entorno socioeconómico. Por ejemplo, las cuestiones a las que los investigadores le conceden mayor importancia en el marco de su actividad científica son: contribuir al avance de conocimiento $(81,3 \%)$, los retos intelectuales $(71,4 \%)$ y tener independencia en la actividad investigadora $(63,8 \%)$. En cambio, la contribución al desarrollo socioeconómico solamente se considera muy importante en el trabajo como investigador por el $31,8 \%$ de los encuestados. Estas preferencias por determinados aspectos del trabajo como investigador muestran la misma lógica en los cuatro perfiles de científicos analizados, ya que no existen diferencias significativas entre los distintos cuadrantes de Stokes.

Figura 1. Forma en que desarrollan la actividad de investigación según cuadrantes de Stokes

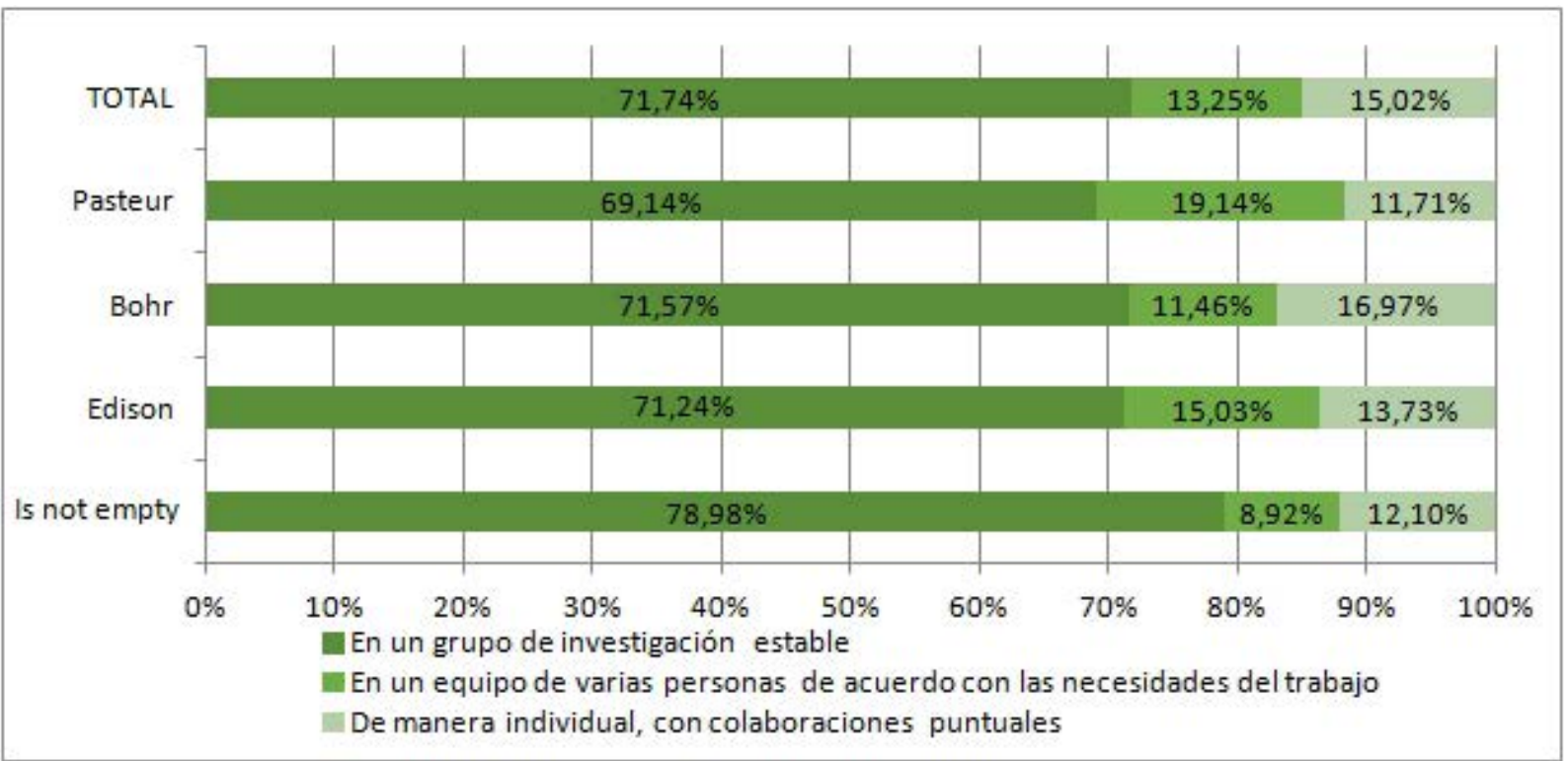

Nota: Los resultados se basan en pruebas bilaterales con un nivel de significación 0.05. 
Figura 2. Grado de importancia concedido a distintos aspectos del trabajo como investigador

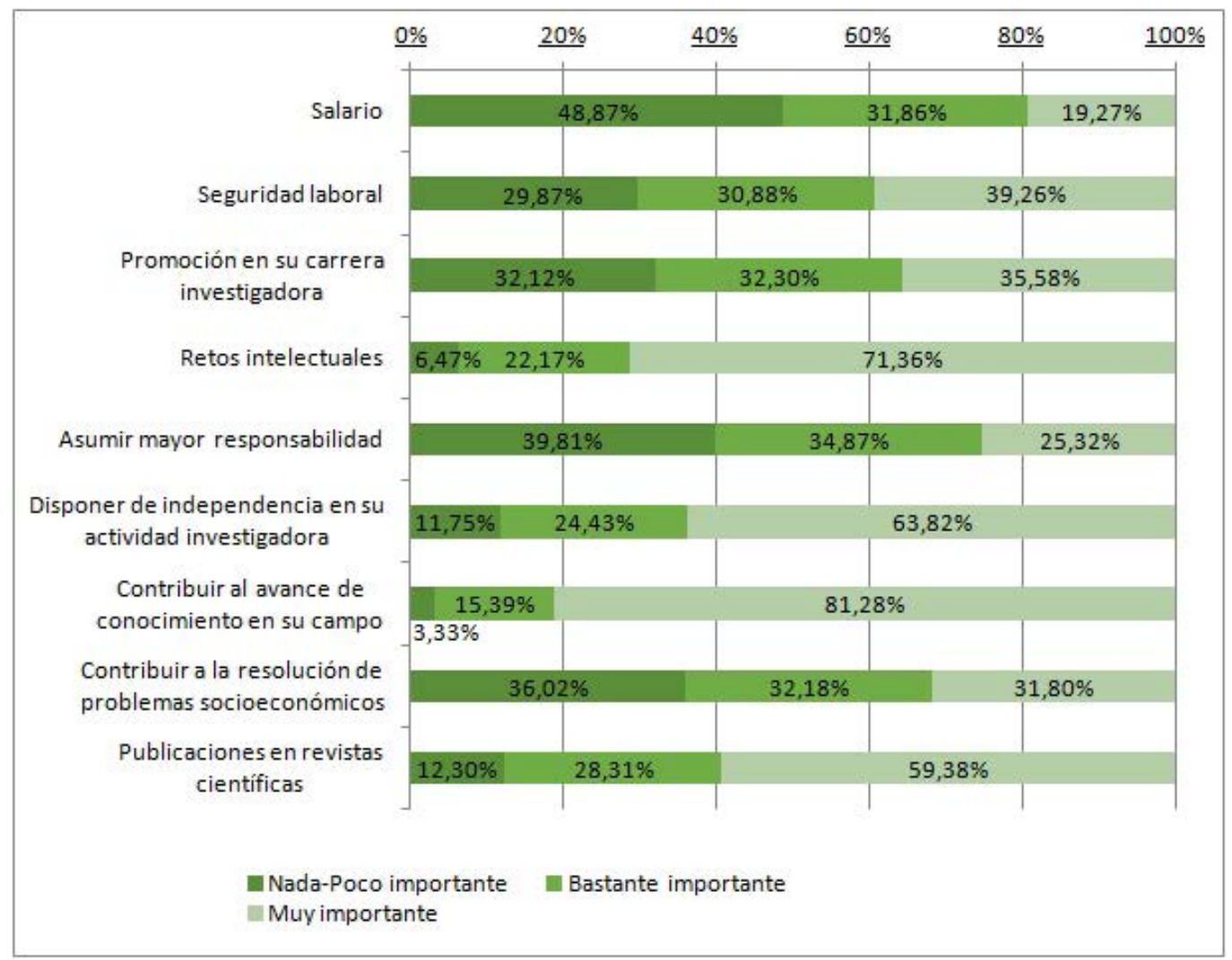

En la misma línea se refleja el análisis del porcentaje medio aproximado de tiempo que dedican los investigadores a las distintas actividades en una semana habitual de trabajo (Figura 3). La actividad que ocupa más de la mitad del tiempo de trabajo es la investigación, con un porcentaje de tiempo medio del $62,3 \%$, seguida de las actividades de gestión que ocupan el 23,38\% de tiempo medio. Mientras tanto, las actividades dirigidas a la relación con otras entidades y a la divulgación de los resultados tienen un peso cuantitativamente menor en la jornada de trabajo, con un $6,45 \%$ y $4,05 \%$ medio de tiempo de trabajo dedicado a estas tareas respectivamente, solamente superior al tiempo medio dedicado a las actividades docencia. Al igual que sucedía cuando se analizaba el grado de importancia concedido a los distintos aspectos del trabajo como investigador, no encontramos diferencias significativas entre los distintos cuadrantes de Stokes a la hora de analizar el porcentaje de tiempo medio dedicado a las distintas actividades en una semana habitual de trabajo.

Cuando se explora, en tercer lugar, la actitud de estos investigadores hacia la vinculación del CSIC con el entorno socioeconómico, se advierte que, pese a que la mitad de los científicos orientan su actividad hacia la comprensión de fenómenos sin implicarse en su aplicación (cuadrante Bohr) y sólo el $22 \%$ de los investigadores se posicionan en el cuadrante de Pasteur ya que buscan también la aplicación de sus conocimientos, los resultados obtenidos en la encuesta revelan que, en general, existe una opinión favorable hacia dicha interacción. En concreto, más del $80 \%$ de científicos están bastante de acuerdo con que el CSIC realice actividades de investigación contratadas por empresas o que se implique en el desarrollo socioeconómico. Sin embargo, sólo cinco de cada diez investigadores están bastante de acuerdo con que el CSIC se implique en la creación de empresas o participe en el capital de empresas. Por otra parte, cuando se analiza la postura de los distintos perfiles de científicos según la orientación de su actividad investigadora (Tabla VII), se advierte que los investigadores situados en los cuadrantes de Edison y Pasteur muestran, en general, una actitud más favorable hacia la realización de este tipo de actividades de transferencia, frente a los investigadores situados en el cuadrante de Bohr que aglutina al $58 \%$ de la comunidad científica. 
Figura 3. Porcentaje de tiempo medio dedicado a distintas actividades en una semana habitual de trabajo

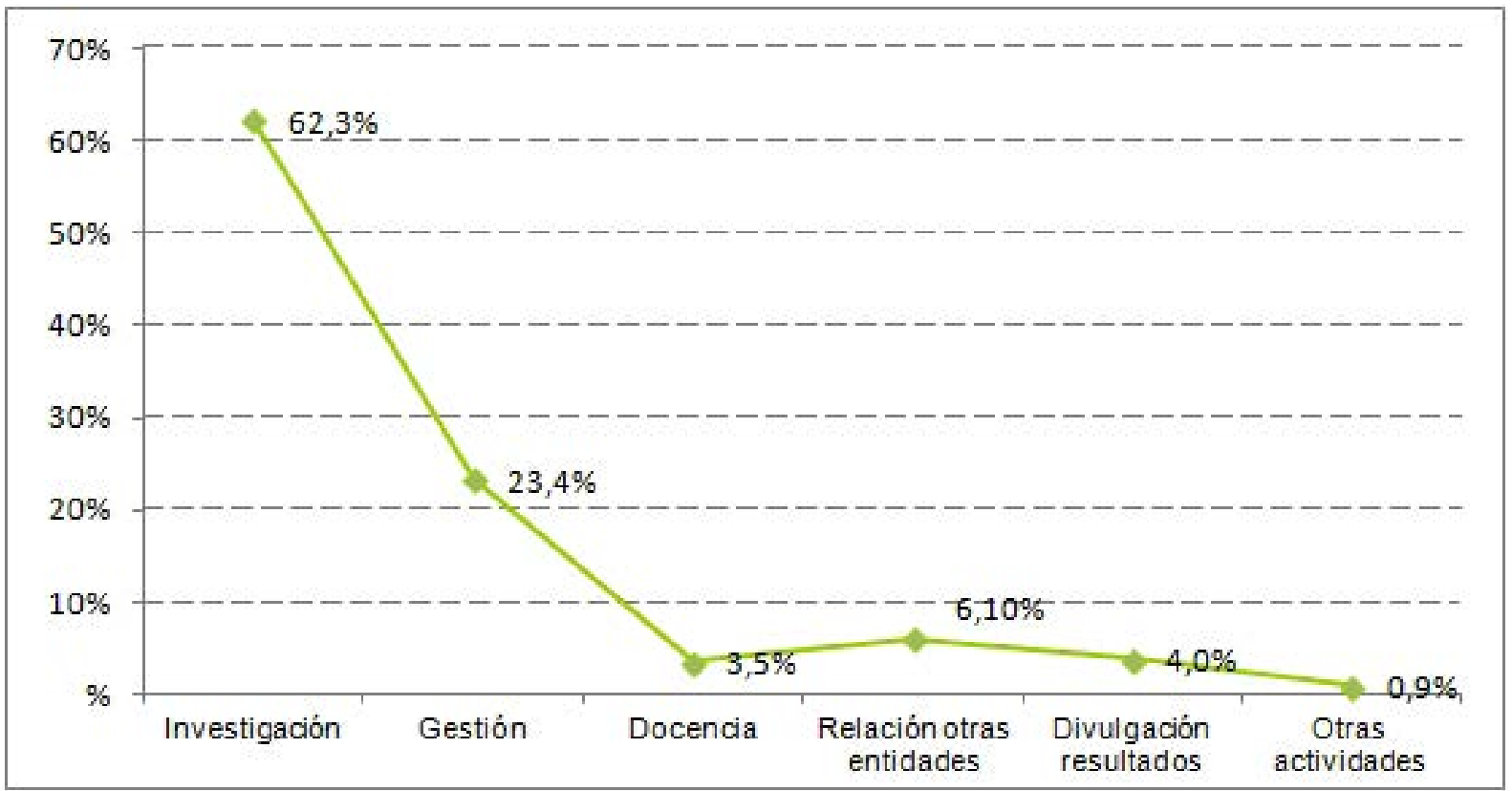

Tabla VII. Grado de acuerdo con que los organismos públicos de investigación realicen las siguientes actividades, según cuadrantes de Stokes

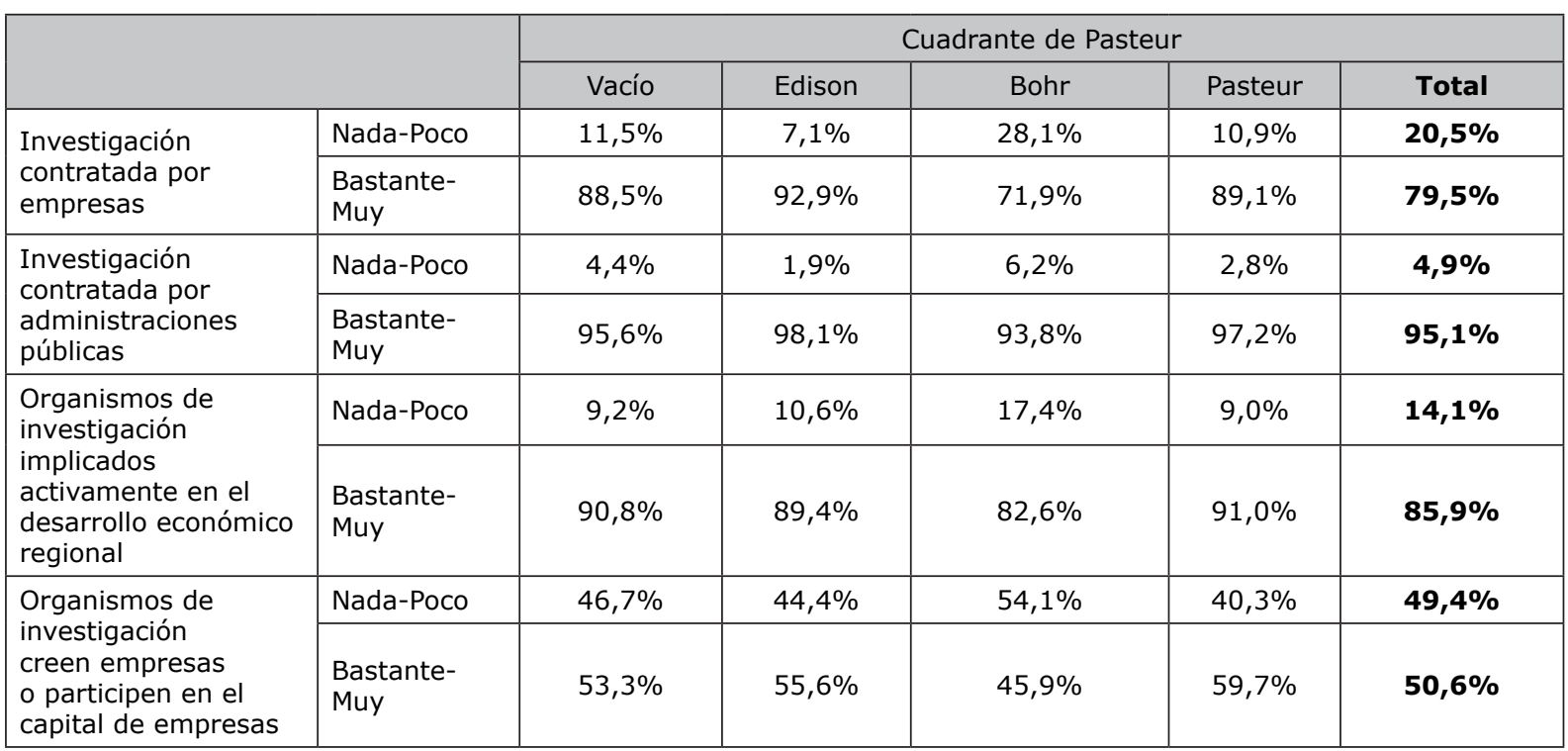

Nota: Los resultados se basan en pruebas bilaterales con un nivel de significación 0.05.

Esto enlaza, finalmente, con el estudio de las motivaciones de los investigadores para mantener vínculos con otras entidades del entorno socioeconómico, teniendo en cuenta, tanto empresas privadas, como organismos de la Administración Pública, organismos internacionales y entidades sin ánimo de lucro. Entre las razones manifestadas por los investigadores están las relacionadas con aspectos que contribuyen a mejorar sus actividades de investigación, bien sea a través de la consecución de financiación adicional o la obtención de información para el desarrollo de sus actuales líneas de investigación o la exploración de otras nuevas, si bien la razón más importante para desarrollar estas relaciones es conseguir becas y oportunidades laborales para estudiantes o doctorandos (Figura 4). Este orden de motivaciones para vin- 
cularse con agentes externos sí varia de forma significativa en función de los distintos perfiles de científicos identificados según su orientación investigadora.

En concreto, como puede observarse en la Figura 4, las diferencias en el nivel de importancia concedido a las distintas motivaciones de los investigadores de los cuadrantes de Edison y Pasteur, frente a los otros dos perfiles de científicos, menos orientados a la aplicación de conocimientos, resultan más sobresalientes los motivos como comprobar la validez y/o aplicación de la investigación que desarrollan, conseguir fondos adicionales, mantenerse informado sobre los temas que interesan a las entidades y acceder a la experiencia de profesionales no académicos.

En definitiva, estos motivos más importantes para establecer relaciones con otras entidades responden a un uso instrumental de las actividades de transferencia. Aunque en principio la actitud es favorable, se puede interpretar que, cuando los investigadores se involucran en estas actividades, en realidad persiguen adaptarlas al contexto en el que trabajan. Guían su actividad investigadora para establecer relaciones con el entorno socioeconómico impulsados por la búsqueda de recursos económicos, si bien el desarrollo de las propias líneas de investigación reflejan prácticas que se encuentran alineadas con los principios de las comunidades científicas de referencia y el sistema de incentivos y recompensas de la ciencia académica tradicional y que, por lo tanto, pueden generar tensiones con sus procesos de relación con entidades ajenas al mundo científico.

\section{CONCLUSIONES}

Este trabajo aplica el modelo teórico de análisis de Stokes (1997) para el análisis de la comunidad científica de un OPI y contribuye así empíricamente a su estudio deteniéndose en tres aspectos fundamentales: primero, qué perfil de investigadores se identifican según la orientación de la actividad científica realizada; segundo, cuál es la actitud de los investigadores hacia la transferencia de conocimiento según los distintos perfiles de investigadores; y, tercero, qué motivaciones conducen a los diferentes perfiles de investigadores a relacionarse con el entorno socioeconómico. Este estudio ofrece, por tanto, implicaciones relevantes para la comunidad científica y los decisores

Figura 4. Motivaciones para establecer relaciones con otras entidades según cuadrantes de Stokes

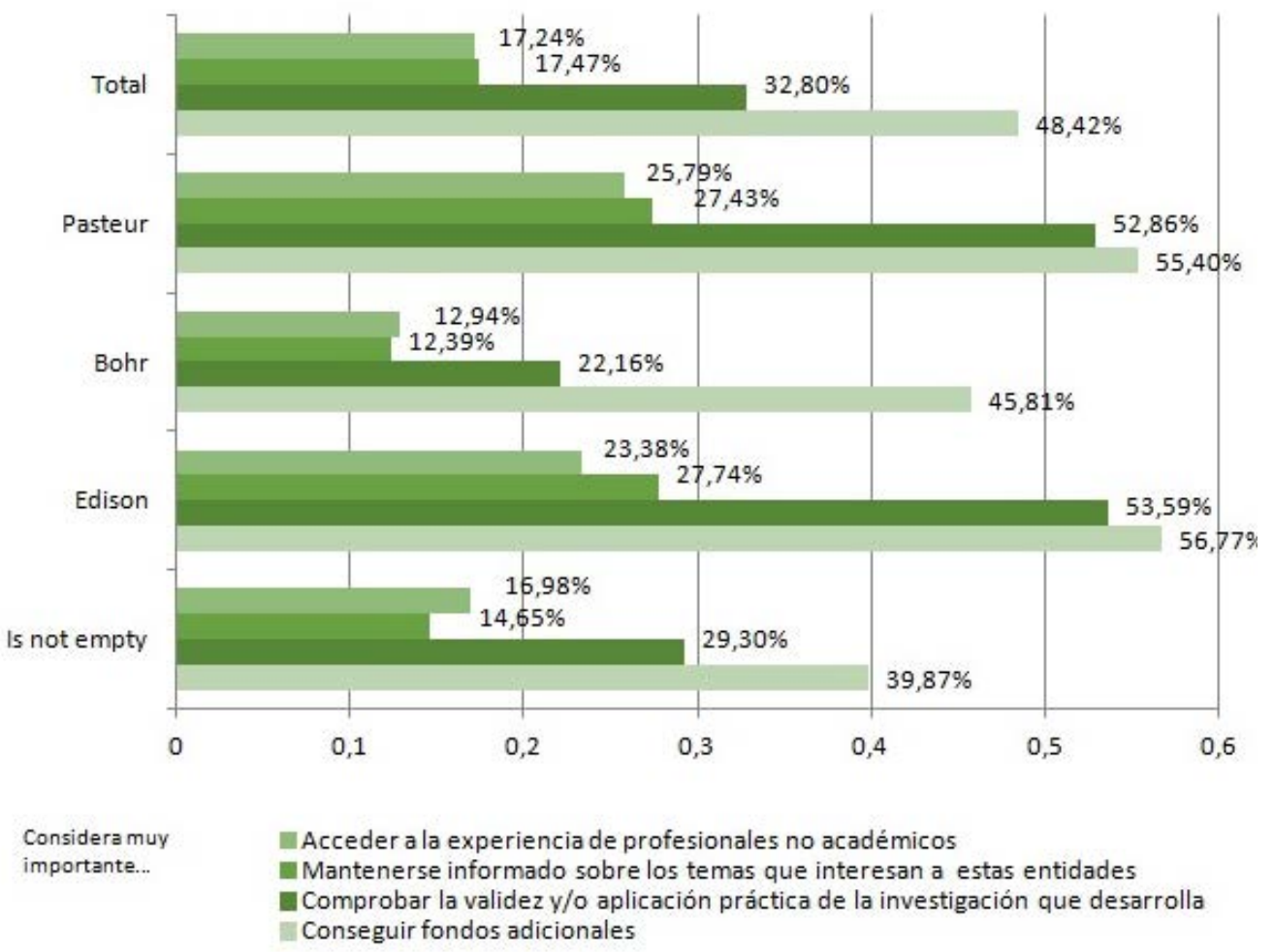

Nota: Los resultados se basan en pruebas bilaterales con un nivel de significación 0.05 . 
públicos de OPIs a fin de trazar un camino sostenible en dirección hacia la evaluación de la actividad investigadora y la transferencia de conocimiento científico.

La dinámica predominante y transversal en toda comunidad científica estudiada se caracteriza fundamentalmente por orientar su actividad investigadora hacia la comprensión de los fenómenos y hechos, frente a una investigación inspirada por la utilización de los conocimientos. Esta dinámica impregna, por tanto, las pautas generales de trabajo de los investigadores del CSIC que reconocen como los aspectos más importantes de su quehacer científico prácticas o valores que se encuentran más alineadas con los principios de la "ciencia académica tradicional" y que, por lo tanto, pueden generar tensiones con el proceso de vinculación con el entorno socioeconómico. Sin embargo, resulta paradójico que los análisis evidencian cómo los investigadores no consideran la vinculación como algo negativo en sí mismo, mostrando una actitud favorable hacia ella, si bien en su trabajo cotidiano, por norma general, predominan aquellas actividades que les permiten consolidarse en su carrera científica, sin olvidar el peso importante que tienen las tareas de gestión en el día a día de la jornada laboral de los investigadores.

Teniendo en cuenta todo lo anterior, puede decirse que aunque los investigadores no consideran la vinculación como algo negativo per se y que incluso muestran una actitud favorable hacia ella, sigue predominando en su escala de "valores" el ethos de la ciencia académica merthoniana, por lo que, en la práctica, dirigen sus esfuerzos hacía aquellas actividades que le permiten afianzarse en este contexto de trabajo. En otras palabras, estos datos sugieren que lo que se busca por parte de la comunidad científica estudiada es adaptar las actividades realizadas con el entorno socioeconómico a su trabajo científico, y no tanto adaptar las potencialidades del trabajo científico al uso práctico y demanda del entorno. Lejos queda todavía en la comunidad científica del CSIC la generalización de esa figura de científico emprendedor que compagina su actividad investigadora tradicional, de contribuir al conocimiento científico y publicar, con el hábito emprendedor basado en la búsqueda de resultados potencialmente comerciales. A esto hay que sumar los impedimentos de gestión que generalmente existen dentro de los OPIs, tanto para investigadores como para empresas, a la hora de colaborar y realizar transferencia de conocimiento y tecnología, con una orientación más aplicada a la resolución de problemas de la sociedad. Generalmente, este tipo de relaciones conllevan un exceso de burocracia por parte de los organismos públicos, que finalmente provoca retrasos en el cumplimiento de objetivos y fechas, y supone un desincentivo, tanto para empresas como para investigadores.
A la vista de estos resultados en este estudio, se pueden derivar diferentes implicaciones a fin de promover la vinculación de la comunidad científica con el entorno socioeconómico. Especialmente, los rectores de la política científica deberían revisar de forma más activa el sistema de incentivos y recompensas de la comunidad científica como eje vertebrador de la actividad profesional de los investigadores e impulsar la consolidación de estos profesionales en la carrera investigadora, de tal manera que promoviera su actividad, también, hacia las demandas del entorno socioeconómico y orientarla así hacia la transferencia de conocimiento científico. De igual modo, resulta fundamental que los OPIs revisen sus formas de gestión para facilitar la actividad investigadora de aquellos investigadores del tipo Edison o Pasteur. Un mayor fomento de la investigación aplicada al entorno socioeconómico o basado en la demanda por parte de los OPIs, a largo plazo les proporcionaría beneficios, tanto en la mejora de su imagen social, como en la posibilidad de un aumento de captación de recursos, lo que les permitirá no depender tanto de las fuentes de financiación pública.

Finalmente, señalar que este ejercicio de análisis realizado sirve para clasificar tendencias en las actitudes y no para observar comportamientos efectivos, aunque en los resultados obtenidos se comprueba que esta clasificación suele ser consistente con las opiniones hacia la transferencias, con las actividades que se realizan con entidades del entorno socioeconómico y con aspectos fundamentales de la organización del trabajo de los investigadores. Teniendo en cuenta esta limitación del estudio, de cara a futuras investigaciones, sería conveniente indagar más en los distintos perfiles de investigadores identificados a través, por ejemplo, de una análisis de correspondencias múltiples o de un análisis cualitativo que permita profundizar en los resultados obtenidos.

\section{AGRADECIMIENTOS}

Los datos de este trabajo proceden de dos Proyectos Intramurales del Consejo Superior de Investigaciones Científicas titulados "Una aproximación del impacto socioeconómico de las actividades del CSIC", dirigidos respectivamente por los Dres. Ignacio Fernández de Lucio y Manuel Fernández Esquinas, y financiados por la Presidencia del CSIC. Las autoras reconocen la aportación al proyecto de los investigadores/as del Instituto de Estudios Sociales Avanzados (IESA-CSIC), especialmente, a Manuel Fernández Esquinas, Elena Espinosa y Leticia Rodríguez, y a investigadores de INGENIO (CSIC-UPV) como Elena Castro, Pablo D'Este, Antonio Gutiérrez, Liney Manjarrés, y Jaider Vega. 


\section{ACKNOWLEDGEMENTS}

The data used in this article have been obtained in the framework of two project of the CSIC entitled: "An approach to the socioeconomic impact of the activities of the CSIC" funded by the Spanish Council for Scientific Research (CSIC) and directed by Ignacio Fernández de Lucio and Manuel Fernández Esquinas, and funded by the CSIC Presidency.

\section{NOTAS}

1. En el presente trabajo entendemos por interacción con el entorno socioeconómico todo tipo de actividad de

\section{REFERENCIAS}

Azagra-Caro, J. M. (2007). What type of faculty member interacts with what type of firm? Some reasons for the delocalisation of university-industry interaction. Technovation, 27 (11), 704-715. https://doi. org/10.1016/j.technovation.2007.05.003

Azagra Caro, J.; Romero de Pablos, A. (2009). Los determinantes institucionales de las patentes del Consejo Superior de Investigaciones: una aproximación histórica y una dialéctica con la Economía. Revista Española de Documentación Científica, 32 (2), 9-33. https://doi. org/10.3989/redc. 2009.2.680

Balaram, P. (2008), Science, invention and Pasteur's quadrant. Current Science, 94 (8), 961-962.

Bekkers, R.; Bodas-Freitas, I.M. (2008). Analysing knowledge transfer channels between universities and industry: To what degree do sectors also matter?. Research Policy, 37 (10), 1837-1853. https://doi. org/10.1016/j.respol.2008.07.007

Bellucci, A.; Pennacchio, L. (2016). University knowledge and firm innovation: evidence from European countries. The Journal of Technology Transfer, 41(4), 730-752. https://doi.org/10.1007/s10961-015-9408-9

Bjerregaard, T. (2010). Industry and academia in convergence: Micro-institutional dimensions of $R \& D$ collaboration. Technovation, 30 (2), 142-153. https:// doi.org/10.1016/j.technovation.2009.11.002

Bonaccorsi, A.; Piccaluga, A. (1994). A theoretical framework for the evaluation of university-industry relationships. $R \& D$ Management, 24 (3), 229-247. https://doi.org/10.1111/j.1467-9310.1994.tb00876.x

Brito Cruz, C.H. (2008). Políticas para C\&T\&I para o Brasil. http://www.ifi.unicamp.br/ brito

Castro-Martínez, E.; Olmos-Peñuela, J.; Fernandez-deLucio, I. (2016). La vinculación Ciencia-Sociedad: Estereotipos y Nuevos Enfoques. Journal of Technology Management \& Innovation, 11 (2), 121-129. https:// doi.org/10.4067/S0718-27242016000200012

Dasgupta, P.; David, P. (1994). Towards a new economics of science. Research Policy, 23 (5), 487-521. https:// doi.org/10.1016/0048-7333(94)01002-1
The authors acknowledge the collaboration of researchers belonging to this project at the Institute for Advanced Social Studies (IESA-CSIC), especially Manuel Fernández Esquinas, Elena Espinosa and Leticia Rodríguez, and researchers at INGENIO (CSIC- UPV) Elena Castro, Pablo D'Este, Antonio Gutiérrez, Liney Manjarrés and Jaider Vega.

transferencia de conocimiento de la comunidad científica con el sector público y/o privado del entorno.

D'Este, P.; Patel, P. (2007). University-industry linkages in the UK: What are the factors underlying the variety of interactions with industry?. Research Policy, 36 (9), 1295-1313. https://doi.org/10.1016/j. respol.2007.05.002

D'Este, P.; Perkmann, M. (2011). Why do academics engage with industry? The entrepreneurial university and individual motivations. Journal Technology Transfer, 36 (3), 316-339. https://doi.org/10.1007/ s10961-010-9153-z

Etzkowitz, H. (1998). The norms of entrepreneurial science: cognitive effects of the new universityindustry linkages. Research Policy, 27 (8), 823-833. https://doi.org/10.1016/S0048-7333(98)00093-6

Etzkowitz, H.; Leydesdorff, L. (2000). The dynamics of innovation: From National Systems and 'Mode 2' to a Triple Helix of university-industry-government relations. Research Policy, 29 (2), 109-123. https:// doi.org/10.1016/S0048-7333(99)00055-4

Fernández Esquinas, M.; Pérez Yruela M.; Merchán Hernández C. (2006). El sistema de incentivos y recompensas en la ciencia pública española. En Sebastián, J.; Muñoz, E. (eds.). Radiografía de la investigación pública en España. Madrid, Biblioteca Nueva.

Foray, D.; Steinmueller, W.E. (2003). On the economics of R\&D and technological collaborations: Insights and results from the project. Economics of Innovation and New Technology, 12 (1), 77-91. https://doi. org/10.1080/10438590303118

Fuentes, C. de; Dutrénit, G. (2012). Best channels of academia-industry interaction for long-term benefit. Research Policy, 41 (9), 1666-1682. https://doi. org/10.1016/j.respol.2012.03.026

Geuna, A. (2001). The Changing Rationale for European University Research Funding: Are There Negative Unintended Consequences?. Journal of Economic Issues, 35 (3), 607-632. https://doi.org/10.1080/002 13624.2001.11506393 
Lee, Y. S. (1998). University-industry collaboration on technology transfer: Views from the ivory tower. Policy Studies Journal, 26 (1), 69-84. https://doi. org/10.1111/j.1541-0072.1998.tb01925.x

Lee, Y.S. (2000). The Sustainability of University-Industry Research Collaboration: An Empirical Assessment. Journal of Technology Transfer, 25 (2), 111-133. https://doi.org/10.1023/A: 1007895322042

Leydesdorff, L. ; Cooke, P. ; Olazaran, M. (2002). Technology transfer in European regions: Introduction to the special issue. The Journal of Technology Transfer, 27 (1), 5-13. https://doi.org/10.1023/A:1013119802068

Merchán-Hernández, C. (2012). Las relaciones de las empresas con las universidades: estrategias y dinámicas del proceso de cooperación a nivel regional. Arbor, 188 (753), 193-209. https://doi.org/10.3989/ arbor.2012.753n1012

Merchán-Hernandez, C.; Valmaseda-Andía, O.; Fernández-Esquinas, M.; (2015). Challenges of connecting science-industry in peripheral regions: researchers' attitudes, organisational and institutional features as determinants. European Planning Studies, 23 (12), 2600-2620. https://doi.org/10.1080/096543 13.2015.1085497

Merton, R.K. (1973). The Sociology of Science, Theoretical and Empirical Investigations. Chicago: University of Chicago Press.

Mowery, D. C.; Sampat, B. N. (2005). The Bayh-Dole Act of 1980 and university-industry technology transfer: A model for other OECD governments?, The Journal of Technology Transfer, 30 (1-2), 115-127. https://doi. org/10.1007/s10961-004-4361-z

Owen-Smith, J. (2003). From separate systems to a hybrid order: Accumulative advantage across public and private science at Research One universities. Research Policy, 32 (6), 1081-1104. https://doi.org/10.1016/ S0048-7333(02)00111-7

Perkmann, M.; Tartari, V.; Mckelvey, M.; Autio, E.; Broström, A.; D’Este, P.; Fini, R.; Geuna, A.; Grimaldi, R.; Hughes, A.; Krabel, S.; Kitson, M.; Llerena, P.; Lissoni, F.; Salter, A.; Sobrero, M. (2013). Academic engagement and commercialisation: A review of the literature on university-industry relations. Research Policy, 42 (2), 423-442. https://doi.org/10.1016/j. respol.2012.09.007

Price, R.H. ; Behrens, T. (2003). Working Pasteuŕ s quadrant: harnessing science and action for community change. American Journal of Community Psychology, 31 (3-4), 219-223. https://doi.org/10.1023/A: 1023950402338

Schartinger, D.; Rammer, C.; Fischer, M.M.; Frohlich, J. (2002). Knowledge interactions between universities and industry in Austria: sectoral patterns and determinants. Research Policy, 31 (3), 303-328. https://doi.org/10.1016/S0048-7333(01)00111-1

Simmons, P. E.; Brunkhorst, H.; Lunetta, V.; Penick, J.; Peterson, J.; Pietrucha, B.; Staver, J. (2005). Developing a research agenda in science education. Journal of Science Education and Technology, 14 (2), 239-252. https://doi.org/10.1007/s10956-005-4424-4

Sousa, W.; Zamudio, M.P.; Souza, D. de. (2009). R\&D Management and the Stokes Diagram: An Exploratory Study. Journal of Technology Management \& Innovation, 4 (4), 95-109.

Stokes, D. E. (1997). Pasteur's Quadrant: basic science and technological innovation. Washington: The Brookings Institution.

Tijssen, R. J. (2006). Universities and industrially relevant science: Towards measurement models and indicators of entrepreneurial orientation. Research Policy, 35 (10), 1569-1585. https://doi.org/10.1016/j. respol.2006.09.025

Tsao, J. Y.; Boyack, K. W.; Coltrin, M. E.; Turnley, J. G.; Gauster, W. B. (2008). Galileo's stream: a framework for understanding knowledge production. Research Policy, 37 (2), 330-352. https://doi.org/10.1016/j. respol.2007.10.004

Valmaseda-Andia, O.; Albizu-Gallastegi, E.; FernándezEsquinas, M.; Fernández-de-Lucio, I. (2015). La relación entre las empresas españolas y el CSIC: motivaciones, mecanismos y beneficios desde la perspectiva empresarial. Revista Española de Documentación Científica, 38 (4): e109, https://doi.org/10.3989/ redc. 2015.4 .1263 